\title{
Supersaturated Calcium Phosphate Rinse vs. Standard of Care for Mitigating Mucositis in Head and Neck Chemoradiation
}

\author{
Justin Brock ${ }^{1}$, Chris Morris ${ }^{1}$, Kathleen Hotze², Brian Pikkula ${ }^{2 *}$ \\ ${ }^{1}$ Katy Center for Oral and Facial Surgery, Katy, TX, USA \\ ${ }^{2}$ Forward Science, Stafford, TX, USA \\ Email: ^bmp@forwardscience.com
}

How to cite this paper: Brock, J., Morris, C., Hotze, K. and Pikkula, B. (2018) Supersaturated Calcium Phosphate Rinse vs. Standard of Care for Mitigating Mucositis in Head and Neck Chemoradiation. Journal of Cancer Therapy, 9, 262-267.

https://doi.org/10.4236/jct.2018.93023

Received: February 6, 2018

Accepted: March 11, 2018

Published: March 14, 2018

Copyright $\odot 2018$ by authors and Scientific Research Publishing Inc. This work is licensed under the Creative Commons Attribution International License (CC BY 4.0).

http://creativecommons.org/licenses/by/4.0/

\begin{abstract}
Introduction: Oral mucositis (OM) can have a significant impact on patients' quality of life, pain and treatment costs. Almost all patients receiving head and neck chemoradiation develop OM. Patients and Methods: Twenty patients were evaluated retrospectively for OM when undergoing head and neck chemoradiation. The control, soda/salt rinse, was compared to a powdered supersaturated calcium phosphate rinse (SSCPR), SalivaMAX. Results: The SSCPR group experienced a delay in the onset of OM, which was not statistically significant, and a marked reduction in peak OM that was statistically significant $(p>0.001)$. Conclusion: This retrospective study demonstrates that a powdered SSCPR is a safe and effective method of oral mucositis mitigation.
\end{abstract}

\section{Keywords}

Oral Mucositis, Health Care Cost, WHO OM Scale

\section{Introduction}

Oral Mucositis (OM), the inflammation of the mucosal membranes that line the inner surfaces of the mouth, is a common side effect of head and neck cancer therapies such as radiation therapy [1] [2]. OM affects up to $100 \%$ of patients with head and neck malignancies receiving radiation treatment and up to $80 \%$ receiving chemotherapy and hematopoietic stem cell transplantation (HSCT) [2]. The degree of inflammation in OM is typically associated with pain and discomfort and can range from erythema to severe ulceration [3]. The burden of $\mathrm{OM}$ on patients is severe enough to cause interruption or dose reduction of can- 
cer therapy, premature treatment cessation [1] [2], and increased healthcare costs [2] [4]. The pain and discomfort of OM can have a significant impact on a patient's nutritional intake, reduced immunity, and overall quality of life [2] [5]. According to a study by Elting et al. [6], clinically significant mucositis will result in a chemotherapy dose reduction in about $23 \%$ of cycles, and commensurately, grade 3 to 4 mucositis will result in a dose reduction in about $28 \%$ of cycles [1].

The goal of cancer therapy is to deliver the full prescribed therapy in a defined timeframe, however $\mathrm{OM}$ is a significant side effect that prevents these efforts. Therefore, prevention and treatment of OM are crucial for patients receiving cancer therapies to ensure compliance and continuation of treatment [1]. Until recently, few effective OM preventive strategies were available. Previous preventive strategies recommended by European Society of Medical Oncology (ESOM) include high quality clinical practice, good oral hygiene, nutritional support, and adequate pain management [2]. Recommendations include experimental therapies, including the use of various cytokines and growth factors, vitamin and mineral supplementation, and cryotherapy. However, there is no consensus on OM treatment and each institution has established their own standard of care with the most common being sodium bicarbonate and sodium chloride (so$\mathrm{da} /$ salt).

Supersaturated calcium phosphate rinses (SSCPR) have been studied and identified as an effective OM treatment for patients with head and neck cancer receiving chemotherapy and radiation therapy. Wasko-Grabowska et al. [7] evaluated supersaturated calcium phosphate rinse compared to standard of care in patients receiving high dose chemotherapy. In all clinical outcomes of patients treated with BEAM chemotherapy, the supersaturated calcium phosphate group scored markedly better in terms of: incidence of oral mucositis, mean number of days with oral mucositis, parental nutrition, and opioid use. Miyamoto et al. [2] evaluated patients that received at least $4500 \mathrm{cGy}$ to the oral cavity using supersaturated calcium phosphate rinse compared to standard supportive care. The supersaturated calcium phosphate group showed marked improvements over the control group in: PEG tube application, in-patient hospitalization, and incremental costs of treatment for oral mucositis. Quinn et al. [8] reviewed thirty studies and found that the majority (24) reported that a supersaturated calcium phosphate rinse was efficacious at reducing the mucositis grade and/or duration as well as the mucositis pain associated with the condition.

Prior to the advent of SSCPR, there have been few effective mucositis treatments and therefore, the current standard of care for mucositis varies per institution. The most common mucositis therapies are "magic mouthwash" whose formulations also vary per institution, meticulous oral care, sodium bicarbonate/sodium chloride rinses, increased hydration, antibacterials such as chlorhexidine, and analgesics including topicals and opioids. SalivaMAX, a powdered SSCPR indicated for the treatment of xerostomia and mucositis, is relatively new 
to the market. This is the first peer reviewed manuscript addressing a new, powdered form of SSCPR in head and neck oncology.

The objective of this study is to evaluate SalivaMAX in the mitigation of oral mucositis in patients undergoing chemoradiation therapy for head and neck cancer.

\section{Patients and Materials}

This retrospective study evaluated noncontemporaneous control and investigational groups. The control group employed a mucositis treatment, sodium bicarbonate and sodium chloride (soda/salt) rinse, which was standard of care at the center at that time prior to the availability of SalivaMAX. Subsequently, the center began using SalivaMAX as their standard of care for mucositis treatment and these patients became the investigational group.

A chart review was carried out screening approximately 80 charts, and the first ten patients from each group who met the inclusion criteria were incorporated in the study. All patients were treated at a regional cancer center.

Inclusion criteria were:

- diagnosis of head and neck cancer with concurrent chemoradiation: fractionated intensity-modulated radiation therapy (IMRT) to 70 Gy with weekly chemotherapy,

- sufficient chart history to analyze oral mucositis (OM) in the population, and

- an average use of the rinse of at least 3 times per day for their respective group.

Exclusion criteria were:

- gaps in chart history for the desired outcomes.

All patients were thoroughly screened weekly for OM using the WHO Oral Mucositis Scale. Recorded for each patient were Onset of OM and Peak OM. Onset of OM describes the number of weeks after treatment is initiated in which OM first presents. The Peak OM outcome is the highest grade of OM that is recorded from any of the weekly assessments for that patient (Table 1).

\section{Results}

While all patients experienced mucositis (Table 2, Table 3), the SSCPR group markedly reduced the proportion of severe mucositis (Grades 3 and 4) $n=1$, showing more incidences of low grade (Grades 1 and 2) mucositis $n=9$. Whereas, the control group had a larger proportion of severe mucositis $n=4$ as compared to the SSCPR group.

Table 4 provides the analysis of this study. The Onset of OM was delayed by a mean of 0.5 weeks for the SSCPR group. However, this outcome was not deemed statistically significant.

For the assessment of Peak OM, there was a statistically significant difference between the control and SSCPR groups ( $p>0.001$, Fisher's exact test) with a Peak OM mean reduction of 0.6 for the SSCPR group. Peak OM for the soda/salt group was 2.4, while the SalivaMAX group was 1.8 . 
Table 1. WHO oral mucositis scale.

\begin{tabular}{cc}
\hline OM Grade & Description \\
\hline Grade 0 & Normal oral mucosa \\
Grade 1 & Soreness/erythema \\
Grade 2 & Soreness/erythema + ulceration + solid foods \\
Grade 3 & Soreness/erythema + ulceration + liquid diet only \\
Grade 4 & Soreness/erythema + ulceration + oral alimentation impossible \\
\hline
\end{tabular}

Table 2. Onset of oral mucositis.

\begin{tabular}{ccc}
\hline Onset & $\begin{array}{c}\text { SSCPR Group } \\
\text { (number of patients) }\end{array}$ & $\begin{array}{c}\text { Control Group } \\
\text { (number of patients) }\end{array}$ \\
\hline Week 1 & 0 & 0 \\
Week 2 & 0 & 1 \\
Week 3 & 4 & 4 \\
Week 4 & 3 & 5 \\
Week 5 & 3 & 0 \\
\hline
\end{tabular}

Table 3. Peak oral mucositis.

\begin{tabular}{ccc}
\hline OM Grade & $\begin{array}{c}\text { SSCPR Group } \\
\text { (number of patients) }\end{array}$ & $\begin{array}{c}\text { Control Group } \\
\text { (number of patients) }\end{array}$ \\
\hline Grade 1 & 3 & 0 \\
Grade 2 & 6 & 6 \\
Grade 3 & 1 & 4 \\
Grade 4 & 0 & 0 \\
\hline
\end{tabular}

Table 4. Analysis of study.

\begin{tabular}{ccc}
\hline & $\begin{array}{c}\text { Onset of OM } \\
\text { Mean }( \pm \text { SD) [weeks] }\end{array}$ & $\begin{array}{c}\text { Peak OM } \\
\text { Mean }( \pm \text { SD) [WHO OM Scale] }\end{array}$ \\
\hline Soda/Salt Rinse & $3.4( \pm 0.70)$ & $2.4( \pm 0.52)$ \\
SalivaMAX & $3.9( \pm 0.86)$ & $1.8( \pm 0.63)$ \\
$p$ & 0.49 & $>0.001$ \\
\hline
\end{tabular}

No adverse reactions for the soda/salt rinse or the SSCPR were reported.

\section{Conclusions}

The use of the supersaturated calcium phosphate rinse, SalivaMAX, is consistent with other peer reviewed literature in the reduction of OM using a SSCPR [2] [8] [9] [10]. Miyamoto et al. [2] found a statistically significant decrease in the incidence of radiation induced oral mucositis in head and neck radiation patients when using a liquid SSCPR. Miyamoto also reported a statistically significant decrease in hospitalization and PEG tube placement. Also reported was a cost 
savings estimate of $\$ 1722$ - $\$ 6917$ due to the reduction of mucositis using SSCPR in this population, which is based on models for incremental costs for treating mucositis of increasing grades. The estimated savings are due primarily to a lack of feeding tubes and a reduced duration of hospitalization. These savings are commensurate with the figures reported by Taylor [4] even after considering the acquisition cost of the SSCPR. Lastly, the use of SSCPR for the treatment of mucositis has been shown to reduce pain and opioid usage [7] [9] [10].

The advantage of a SSCPR among other drug interventions is the non-systemic nature of SSCPRs. After the powder is mixed with water, the supersaturated calcium phosphate solution is rinsed in the mouth and expectorated. When using a SSCPR, patients undergoing chemoradiation are not subjected to another chemical burden. Additionally, there are no anticipated side effects and no known drug interactions.

While a precise quantification of a reduction in Peak OM is difficult to extrapolate because of the ordinal nature of the data, the takeaway from the assessment of Peak OM shows that SSCPR reduced the mucositis the patient experiences to a statistically significant level. We anticipated a statistically significant delay of the onset of mucositis, which did not materialize. A possible reason could be the discretization of sampling OM to a weekly assessment may have led to an error in determining when OM first started. A more accurate method would have been to assess OM daily or at least bi-weekly.

A shortcoming of this study was the inherent limitations of a retrospective study which do not have the planned foresight to measure and record other outcomes. Gaps in chart history for all the desired outcomes would effectively rule out inclusion of the majority of the patients for this retrospective study, limiting the sample size. Increasing the sample size and adding assessments such as $\mathrm{pH}$ neutralization, total parenteral nutrition, more frequent $\mathrm{OM}$ reporting, a Quality of Life assessment, patient feedback, duration of hospitalization, and pain/opioid medication usage would provide a better overall clinical depiction of the patient; a prospective study incorporating the above is being implemented.

This retrospective study demonstrates that a powdered SSCPR is a safe and effective method of oral mucositis management. SalivaMAX shows promise in reducing the dangerous and costly side effects of OM and improving patient's quality of life. More research is indicated with well-designed studies and with a larger patient population.

\section{References}

[1] Bensinger, W., Schubert, M., Ang, K., et al. (2008) NCCN Task Force Report: Prevention and Management of Mucositis in Cancer Care. Journal of the National Comprehensive Cancer Network, 6, S1-S21.

[2] Miyamoto, C., Wobb, J., Micaily, B., Li, S. and Achary, M. (2012) A Retrospective Match Controlled Study of Supersaturated Calcium Phosphate Oral Rinse vs. Supportive Care for Radiation Induced Oral Mucositis. Journal of Cancer Therapy, 3, 630-636. https://doi.org/10.4236/jct.2012.35081

[3] Sonis, S.T., Oster, G., Fuchs, H., et al. (2001) Oral Mucositis and the Clinical and 
Economic Outcomes of Hematopoietic Stem-Cell Transplantation. Journal of Clinical Oncology, 19, 2201-2205. https://doi.org/10.1200/JCO.2001.19.8.2201

[4] Taylor, D.W. (2013) A Business Impact Study of the Use of a Supersaturated Calcium Phosphate Oral Rinse (SCPOR) in the Prevention and Treatment of Oral Mucositis. Clinical Medicine and Diagnostics, 3, 82-87.

[5] Trotti, A., Belim, L.A., Epstein, J.B., et al. (2003) Mucositis Incidence, Severity, and Associated Outcomes in Patients with Head and Neck Cancer Receiving Radiotherapy with or without Chemotherapy: A Systematic Literature Review. Radiotherapy and Oncology, 66, 253-262. https://doi.org/10.1016/S0167-8140(02)00404-8

[6] Elting, L.S., Cooksley, C., Chambers, M., et al. (2003) The Burdens of Cancer Therapy: Clinical and Economic Outcomes of Chemotherapy-Induced Mucositis. Cancer, 98, 1531-1539. https://doi.org/10.1002/cncr.11671

[7] Wasko-Grabowska, A., Rzepecki, P., Oborska, S., et al. (2011) Efficiency of Supersaturated Calcium Phosphate Mouth Rinse Treatment in Patients Receiving High-Dose Melphalan or BEAM Prior to Autologous Blood Stem Cell Transplantation. Transplantation Proceedings, 43, 3111-3113.

https://doi.org/10.1016/j.transproceed.2011.08.053

[8] Quinn, B. (2013) Efficacy of a Supersaturated Calcium Phosphate Oral Rinse for the Prevention and Treatment of Oral Mucositis in Patients Receiving High-Dose Cancer Therapy: A Review of Current Data. European Journal of Cancer Care, 22, 64-579. https://doi.org/10.1111/ecc.12073

[9] Papas, A.S., Clark, R.E., Martuscelli, G., et al. (2003) A Prospective, Randomized Trial for the Prevention of Mucositis in Patients Undergoing Hematopoietic Stem Cell Transplantation. Bone Marrow Transplantation, 31, 705-713. https://doi.org/10.1038/sj.bmt.1703870

[10] Markiewicz, M., Dzierzak-Mietla, M., Frankiewicz, A., et al. (2012) Treating Oral Mucositis with a Supersaturated Calcium Phosphate Rinse: Comparison with Control in Patients Undergoing Allogeneic Hematopoietic Stem Cell Transplantation. Support Care in Cancer, 20, 2223-2229. https://doi.org/10.1007/s00520-012-1489-5 